Revue des patrimoines

\title{
Une série des femmes illustres ou femmes fortes par Guy François (1578 ?-1650)
}

\section{Marie-Félicie Perez et Bruno Saunier}

\section{(2) OpenEdition}

\section{Journals}

Édition électronique

URL : http://journals.openedition.org/insitu/4514

DOI : 10.4000/insitu.4514

ISSN : 1630-7305

Éditeur

Ministère de la culture

Référence électronique

Marie-Félicie Perez et Bruno Saunier, « Une série des femmes illustres ou femmes fortes par Guy François (1578 ?-1650) », In Situ [En ligne], 10 | 2009, mis en ligne le 19 mai 2009, consulté le 01 mai 2019. URL : http://journals.openedition.org/insitu/4514 ; DOI : 10.4000/insitu.4514

Ce document a été généré automatiquement le 1 mai 2019.

\section{(c) $($ i) $(9)$}

In Situ Revues des patrimoines est mis à disposition selon les termes de la licence Creative Commons Attribution - Pas d'Utilisation Commerciale - Pas de Modification 4.0 International. 


\title{
Une série des femmes illustres ou femmes fortes par Guy François (1578?-1650)
}

\author{
Marie-Félicie Perez et Bruno Saunier
}

1 Guy François est connu comme le principal peintre religieux de la première moitié du XVII siècle du Velay et de l'Auvergne mais également du Languedoc. Il a fait l'objet d'une thèse de doctorat en 1968 soutenue par Marie-Félicie Perez et d'une exposition monographique organisée en 1974 par Roger Gounot au musée Crozatier du Puy-en-Velay et au musée d'Art et d'industrie de Saint-Étienne ${ }^{1}$. Depuis cette date, de nombreuses œuvres - tableaux de retable mais également œuvres de dévotion privée, décors fixes et dessins - sont apparues. Cet ensemble d'environ cent articles, pour partie encore inédit, sera publié dans la monographie et le catalogue raisonné de l'œuvre de Guy François établi par Bruno Saunier. Cet artiste est encore peu représenté dans les collections des musées. À part une salle créé depuis 1974 au musée Crozatier du Puy-en-Velay à partir de dépôts et d'acquisitions judicieuses, le peintre ne figure dans aucun autre musée d'Auvergne. Au Louvre, Guy François est représenté par une belle Sainte Madeleine caravagesque. L'essentiel de son œuvre est encore in situ, soit dans des églises soit dans des propriétés privées difficilement accessibles. Cependant, comme les archives le laissaient pressentir, Guy François n'est pas uniquement peintre d'œuvres religieuses. Outre des portraits dont deux au moins sont connus, il a peint une série des «Femmes illustres », encore inédite et conservée dans une propriété proche du Puy-en-Velay.

2 Cet ensemble éclaire d'un jour nouveau la production de Guy François. En même temps, cette série vient s'ajouter à plusieurs autres sur ce thème particulièrement en faveur dans l'art français de la première moitié du XVII ${ }^{\mathrm{e}}$ siècle. 


\section{Le thème des Femmes illustres ou Femmes fortes dans l'art et la littérature du xvii ${ }^{\mathrm{e}}$ siècle}

3 La première moitié du XVII ${ }^{\mathrm{e}}$ siècle a connu dans l'art et la littérature un développement sans précédent du thème des Femmes illustres.

4 Peuvent être en effet cités les tableaux ornant le cabinet de la reine au château de Richelieu, peints pour le cardinal par Nicolas Prévost (1604-1670), ceux réalisés par Simon Vouet vers 1645-1646 au Palais Cardinal à Paris pour l'appartement de la régente Anne d'Autriche, ceux encore dus au pinceau de Charles Poerson, également vers 1645, encastrés dans les lambris de l'appartement de Madame de La Meilleraye à l'Arsenal de Paris, sans doute la série la plus importante (quinze figures). À la même époque, Claude Vignon donnait des modèles pour illustrer La Galerie des Femmes fortes publiée en 1647 par le jésuite Pierre Le Moyne, œuvres gravées par Gilles Rousselet et Abraham Bosse et reprises par la suite dans plusieurs domaines des arts décoratifs. Il convient également de mentionner les tentures de tapisserie créées dans les ateliers parisiens et de La Marche d'après des modèles de Charles Poerson, déjà cité, mais également d'Isaac Moillon et diffusées à de nombreux exemplaires² ${ }^{2}$.

5 Rappelons enfin le thème parallèle développé dans l'art français de la première moitié du $\mathrm{XVII}^{\mathrm{e}}$ siècle, et particulièrement une fois encore dans la tapisserie, celui de l'Histoire d'Artémise ${ }^{3}$. On sait combien le développement du tissage de tentures de tapisserie sur le thème des «Femmes illustres» ou celui d'Artémise dans les ateliers français est lié à l'histoire politique de la France dans la première moitié du XVII e siècle avec la succession de deux périodes de régence conduites par deux femmes, Marie de Médicis et Anne d'Autriche, épouses puis veuves de deux rois et mères de deux jeunes rois mineurs, comme l'était Artémise, veuve du roi Mausole et mère du jeune Lygdamis, femmes illustres par leur courage face à l'adversité pour préserver l'héritage de leur enfant.

6 Le thème des Femmes illustres ou des Femmes fortes se retrouve également dans la littérature où il se développe au cours de la première moitié $\mathrm{du} \mathrm{XVII}{ }^{\mathrm{e}}$ siècle mais, semble$\mathrm{t}$-il, moins en relation avec le contexte politique des régences qu'accompagnent les différentes frondes contre le pouvoir. Sans faire le recensement des textes parus sur le sujet, citons, néanmoins, le Théâtre françois des seigneurs et des dames illustres du père François Dinet, la Gallerie des dames illustres de François de Grenaille, ouvrages parus en 1642, les Femmes illustres de Madeleine et Georges de Scudéry, publié en 1642 et 1644, La femme héroïque du père Jacques Du Bosc, de 1645, les Éloges des douze dames illustres grecques, romaines et françoises dépeintes dans l'alcôve de la reine, ouvrage anonyme paru en 1646 et, enfin, sans doute le plus connu des historiens de l'art en raison des illustrations d'après Claude Vignon, La Gallerie des femmes fortes du père Pierre Le Moyne, publié en 1647. On renverra à l'article très documenté de Catherine Pascal sur ce thème des recueils de femmes illustres au XVII siècle ${ }^{4}$. L'auteur insiste sur l'évolution du statut de la femme depuis la Renaissance, à la fois «créature diabolique, agent de Satan, incarnation du péché originel » mais également, depuis le concile de Trente et la place grandissante accordée à la Vierge dans le culte catholique, « médiatrice de la foi ».

7 Ce thème des Femmes illustres ou Femmes fortes est donc en partie lié à la ContreRéforme catholique, comme l'est celui de Marie Madeleine pénitente, si cher aux peintres du XVII ${ }^{e}$ siècle. 


\section{La série des Femmes illustres d'une propriété de la Haute-Loire}

8 Signalée en 1984 dans une propriété des environs du Puy-en-Velay, cette série de « Femmes illustres » n'a jamais été étudiée même si Léon Giron, conservateur du musée Crozatier à la fin du XIX ${ }^{\mathrm{e}}$ siècle, la mentionne en l'attribuant à Jean François le fils de Guy, lui-même peintre, avec une médiocre appréciation, mais en la rapprochant des illustrations de l'ouvrage du père Le Moyne ${ }^{5}$. Il s'agit de six tableaux, de moyen format, mesurant pour chacun d'entre eux $107 \mathrm{~cm}$ de haut par $83 \mathrm{~cm}$ de large, sauf la toile figurant Judith, légèrement plus petite puisqu'elle mesure seulement $99,5 \mathrm{~cm}$ par $81 \mathrm{~cm}$. Chaque toile est bordée d'un cadre en bois peint mouluré caractéristique de la première moitié du XVII ${ }^{e}$ siècle. Mais il est impossible de savoir s'il s'agit des cadres d'origine ou si les toiles étaient enchâssées dans un décor fixe.

\section{L'artiste a figuré les femmes illustres suivantes :}

$91^{\circ}$ La reine Zénobie, représentée en reine guerrière, casquée, couronnée et vêtue d'un manteau doublé d'hermine, prisonnière, les mains entravées, et enchaînée. Elle est accompagnée d'une suivante et d'un bourreau qui tient la chaîne (fig. $\mathbf{n}^{\circ} \mathbf{1}$ ).

$102^{\circ}$ Panthée qui découvre le corps de son mari Abradate, roi de Suse, mort au combat, et se suicide en s'enfonçant une épée dans la poitrine (fig. $\mathbf{n}^{\circ} 2$ ). 


\section{Figure 1}

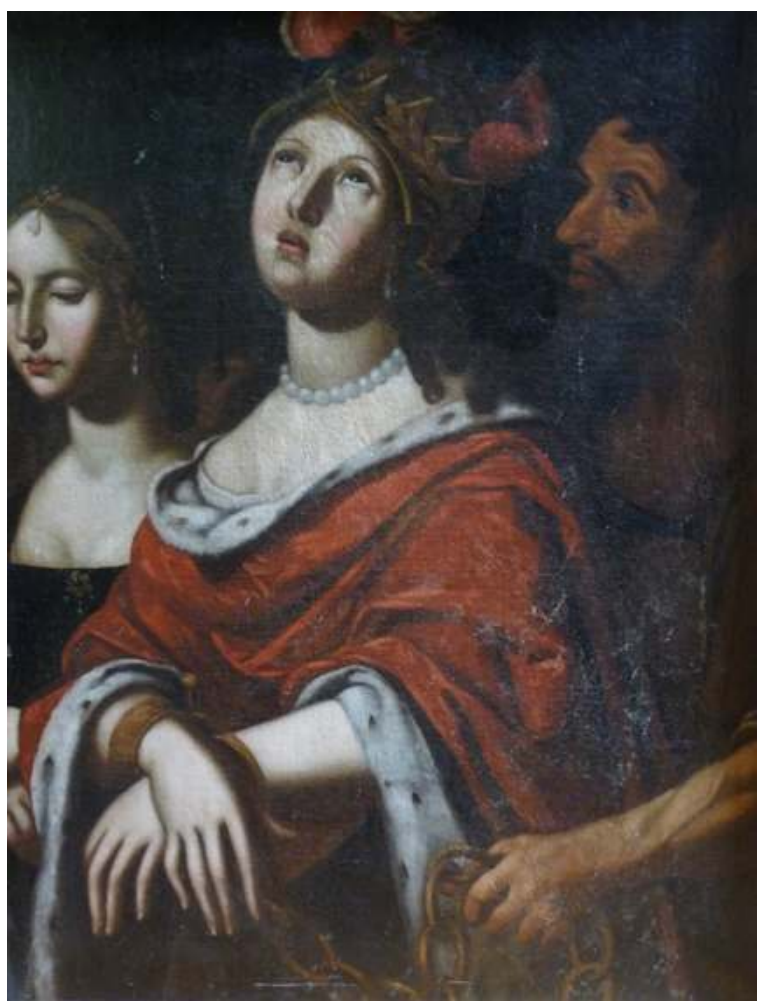

Guy François et atelier, la reine Zénobie, huile sur toile, $107 \mathrm{~cm}$ x $83 \mathrm{~cm}$, Haute-Loire, collection privée. Bruno Saunier, 2008

(c) Bruno Saunier. 


\section{Figure 2}

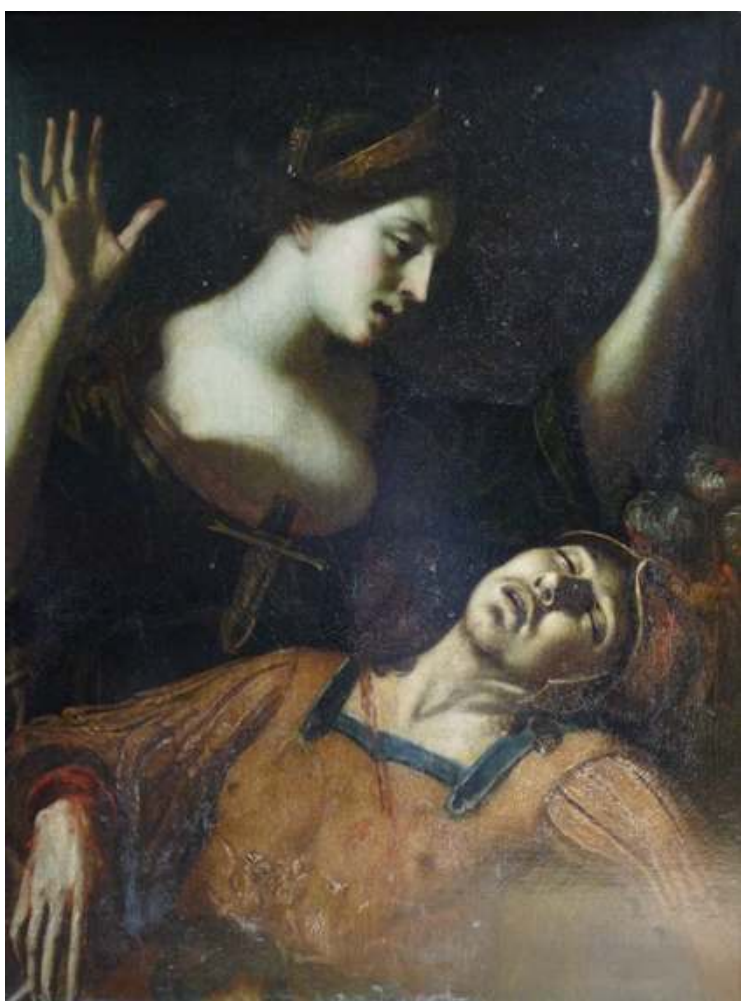

Guy François, Panthée, huile sur toile, $107 \mathrm{~cm}$ x 83 cm, Haute-Loire, collection privée. Bruno Saunier 2008.

(c) Bruno Saunier.

$113^{\circ}$ Lucrèce qui, violée par Sextus, fils de Tarquin le Superbe, se suicide en se perçant le cœur d'un poignard (fig. $\mathbf{n}^{\circ} 3$ ).

$124^{\circ}$ Porcia qui, à la suite de la défaite de son mari, Brutus, fils adoptif de César, en 42 avant J.-C. à Philippes, se suicide en avalant des charbons ardents (fig. $\mathbf{n}^{\circ} \mathbf{4}$ ). 


\section{Figure 3}

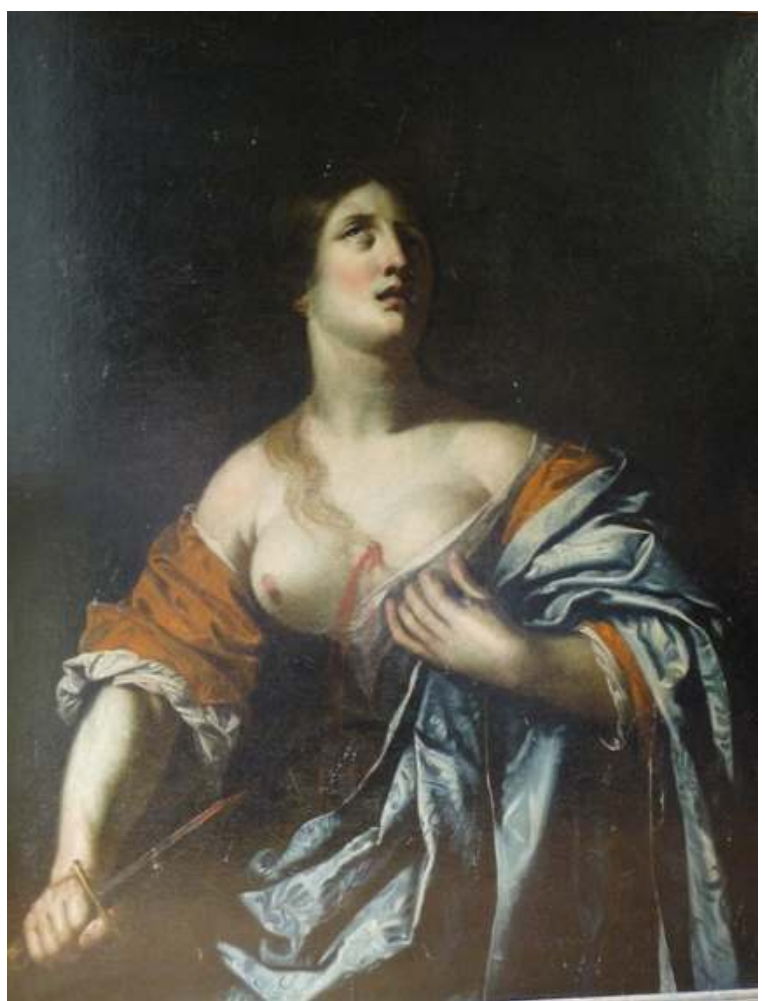

Guy François (d'après Guido Reni), Lucrèce, huile sur toile, $107 \mathrm{~cm} \times 83 \mathrm{~cm}$, Haute-Loire, collection privée. Bruno Saunier, 2008.

(c) Bruno Saunier. 


\section{Figure 4}

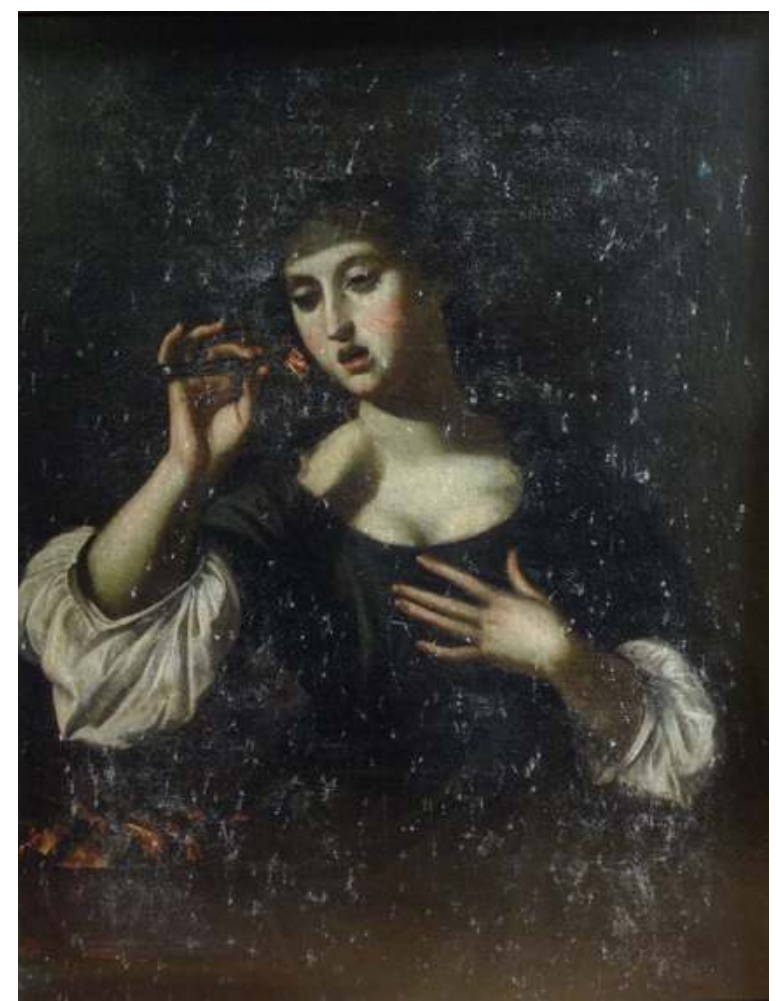

Guy François, Porcia, huile sur toile, $107 \mathrm{~cm}$ x 83 cm, Haute-Loire, collection privée. Bruno Saunier, 2008.

(c) Bruno Saunier.

$135^{\circ}$ Judith, jeune héroïne juive de Judée, est représentée l'épée dans la main droite et tenant la tête du général de Nabuchodonosor, Holopherne, qu'elle vient de décapiter dans son sommeil (fig. $\mathbf{n}^{\circ}$ ).

$146^{\circ}$ Une reine, coiffée d'un diadème et vêtue d'un riche manteau doublé d'hermine, tenant une pomme dans sa main gauche et une torche enflammée dans sa main droite. Elle est représentée devant une tenture verte découvrant une ville en flammes. Sans doute s'agitil de Marie Stuart, reine d'Écosse. Elle est parfois figurée dans les séries des «Femmes illustres » (fig. $\mathbf{n}^{\circ}$ 6). 


\section{Figure 5}

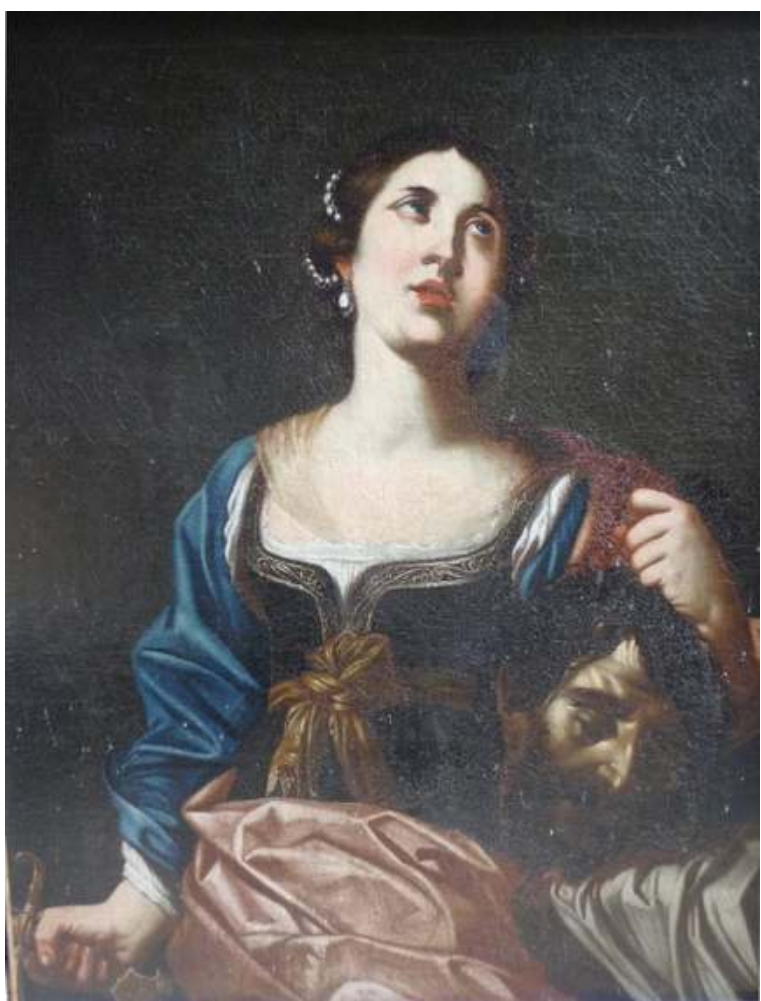

Atelier de Guy François, Judith, huile sur toile, $99,5 \mathrm{~cm}$ x 81 cm, Haute-Loire, collection privée. Bruno Saunier, 2008.

(c) Bruno Saunier. 
Figure 6

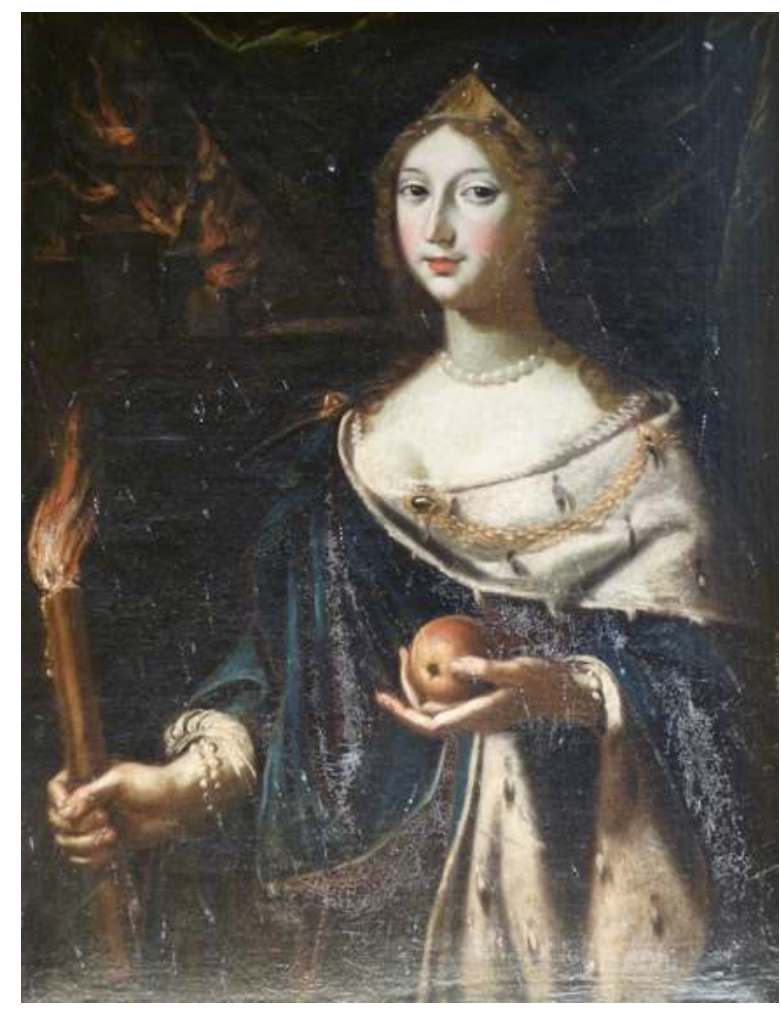

Guy François, Marie Stuart ( ?), huile sur toile, $107 \mathrm{~cm}$ x $83 \mathrm{~cm}$, Haute-Loire, collection privée. Bruno Saunier, 2008.

(c) Bruno Saunier.

15 La logique ayant présidé au choix de ces six héroïnes n'apparaît pas d'emblée, même si ce nombre de six tableaux permet, a priori, de construire une suite équilibrée. En effet, deux femmes barbares de la Grèce antique, Panthée et Zénobie, accompagnent deux femmes de l'histoire romaine, Lucrèce et Porcia, mais il manque une femme juive de l'Ancien Testament et Marie Stuart, femme forte de l'époque moderne, est également isolée. Cependant, pourraient être associés à cette série deux autres tableaux, l'un, vraisemblablement perdu et l'autre, conservé dans une autre branche de la famille propriétaire mais que nous n'avons pas vu. Le premier représentait Jahel, une héroïne de l'Ancien Testament et il est mentionné dans l'inventaire après décès du frère cadet de Guy François, Claude, marchand chaudronnier: À la Salle A esté trouvé un grand tableau représentant Énée et d'autres (?) avec une sibylle, une saincte Agate et une Jael toille d'empereur'. La cohérence se renforcerait, Jahel venant compléter Judith mais il manque encore le pendant de la présumée Marie Stuart.

Ces femmes illustres ne sont pas toutes vertueuses ni fidèles, Marie Stuart, dont la vie est encore précisément connue au XVII ${ }^{\mathrm{e}}$ siècle, ayant eu une liaison avec Jacques Hepburn, quatrième comte de Bothwell, tout en étant encore mariée à son deuxième époux, Henry Stuart. En revanche, elles font toute preuve d'un grand courage face à l'adversité et meurent avec détermination.

17 L'artiste a adopté un cadrage serré, représentant chaque héroïne à mi-corps et occupant la totalité de l'espace. Un fond neutre est adopté pour chacune des toiles, sauf pour celle représentant Marie Stuart dont l'arrière-plan est occupé par une tenture verte ouvrant 
sur une ville en flammes. Chacune des femmes est représentée de face ou légèrement de trois-quarts, une partie du visage laissée dans l'ombre. Variété des attitudes et des vêtements qui enlève toute monotonie à cette série de six tableaux. On constate également une certaine diversité dans le traitement des tissus et le modelé des chairs. Panthée, Porcia, Lucrèce et Marie Stuart semblent traitées avec vigueur, la touche du pinceau étant parfois rapide, allusive même, tandis que Zénobie est représentée avec quelque maladresse et que Judith est d'une facture plus sèche, les ombres étant beaucoup plus appuyées que dans les tableaux précédents. En même temps, si les visages ovales de Panthée, Zénobie, Porcia et Marie Stuart, avec leur bouche menue et la partie laissée dans l'ombre, sont tout à fait caractéristiques du style de Guy François, ceux de Judith et de Lucrèce s'éloignent davantage de ses créations. On relève dans les quatre premiers tableaux cités d'autres traits propres au style de l'artiste: l'éloquence des gestes des mains, souvent très longues, les yeux révulsés et les bouches ouvertes. Le cadavre d'Adrabate sur les genoux de Panthée est une sorte de réplique païenne des Christs morts des Vierges de pitié de l'artiste.

Cependant, malgré certains traits communs aux six tableaux, l'impression générale est celle d'un manque d'homogénéité et d'une réalisation par plusieurs mains. Une première explication peut être donnée à travers le cas de Lucrèce. En effet, l'artiste s'est inspiré d'une œuvre de même sujet due à Guido Reni et dont il existe au moins deux versions autographes, l'une dans une collection privée à New York, datée 1632, et l'autre dans une collection privée à Modène, plus de nombreuses copies et variantes d'atelier'. L'attitude générale est la même dans l'œuvre de Guido Reni et dans celle de Guy François : même regard tourné vers le haut, même geste de la main gauche dénudant la poitrine, même vêtement aux plis amples. Mais Guy François adopte une position différente pour le bras droit de sa Lucrèce: alors que dans le tableau de Guido Reni elle s'apprête à se poignarder, dans celui de notre peintre le geste fatal vient d'avoir lieu et l'épouse de Tarquin Collatin, dans un geste théatral, retire le poignard ensanglanté de sa poitrine de laquelle jaillit un flot de sang. De même, à la différence de Guido Reni, Guy François adopte pour la tête de Lucrèce une position moins penchée vers la droite du tableau, plus statique.

La Judith semble, elle aussi, dériver d'un autre tableau dont nous n'avons pas pu identifier la source. Le thème, très caravagesque, a été traité à de nombreuses reprises dans toute la peinture occidentale de la première moitié du XVII ${ }^{e}$ siècle. Cependant, nous aimerions voir dans cette Judith conservée en Haute-Loire une copie d'un tableau de Massimo Stanzione ou de son entourage. Les traits beaucoup plus appuyés que dans les autres tableaux de la série, plus systématiques, et le côté appliqué du pinceau laissent penser qu'il s'agit d'une copie et non, à la différence du précédent, d'une création inspirée d'une autre œuvre.

\section{Quelle place pour cette série de tableaux dans l'art de Guy François?}

Sans reprendre les remarques exprimées précédemment, nous voudrions insister sur l'absence d'homogénéité de ces six tableaux. Si les uns sont peints avec vigueur, d'autres paraissent exécutés avec plus de mollesse et l'un présente une facture très sèche. Guy François est capable de peindre des tableaux d'une grande qualité d'exécution avec des modelés délicats et un rendu des matières d'une grande vérité, notamment dans les 
plumes et pelages des animaux, les textiles et les terres cuites de ses natures mortes. L'une de ses dernières œuvres datées, la Présentation au Temple de l'église Saint-Julien de Tournon-sur-Rhône, dans l'Ardèche, de 1645, témoigne de ce savoir-faire et, plus généralement, de son talent. Il est vrai que ses deux dernières œuvres datées avec précision, postérieures d'une année à la précédente, l'Adoration des Bergers et l'Adoration des Mages, conservées dans l'église de Pontgibaud (Puy-de-Dôme) mais provenant initialement de la chartreuse de Port-Sainte-Marie non loin de là, sont de facture plus sèche. Ce sont cependant des répliques de tableaux antérieurs de même sujet.

Guy François meurt en 1650 et nous pensons pouvoir situer la série des «Femmes illustres » peu avant cette date. Il s'agit même certainement des dernières créations de l'artiste. En 1650, Guy François est âgé de soixante-douze ans, si l'on accepte une date de naissance en $1578^{8}$. Il fait son testament le 5 octobre et meurt vraisemblablement quelques jours après 9 . Le notaire François Maréchal qui procède à l'inventaire de ses biens le dit «dans son lit malade». À cette époque, Guy François laisse, outre une fortune relativement importante pour un peintre, un atelier florissant dans lequel il a formé son fils Jean qui prendra la suite de son père mais sans en avoir le talent et son gendre, Jean Solvain, également peintre et issu lui-même d'une famille de peintres.

On perçoit dans certaines œuvres de la maturité de Guy l'intervention d'autres mains. C'est le cas de la série des « Femmes illustres » et il ne serait pas étonnant de voir dans ces œuvres la participation de l'atelier, sans doute celle de Jean François que son père voulait vraisemblablement «lancer» mais aussi peut-être celle de son gendre, Jean Solvain, certainement le plus doué des deux. Si Panthée, Lucrèce, Porcia et Marie Stuart reviennent assurément à Guy, Zénobie est de moins bonne qualité et Judith est une copie au traitement sec. Sans certitude, on peut imaginer que Guy François aurait reçu cette commande mais que son grand âge (puis son décès) ne lui aurait pas permis de la terminer lui-même, l'atelier achevant alors les tableaux.

Il convient de mettre la série des «Femmes fortes » en relation avec un autre ensemble de toiles attribuées avec une quasi-certitude à Guy François; les six représentations de femmes du château d'Aulteribe à Sermentizon (Puy-de-Dôme), sans doute un ensemble provenant d'un décor aujourd'hui démembré et dont elles auraient pu constituer la partie haute $^{10}$. L'iconographie de cette série est plus mystérieuse que celle des «Femmes fortes ", puisque sont représentées trois femmes avec des sceptres et des couronnes, sans doute des reines, une musicienne et deux femmes avec des nourrissons (l'un étant mort) (fig. $\mathbf{n}^{\circ} \mathbf{7}, \mathbf{n}^{\circ} \mathbf{8}$ ). On pourrait aussi voir dans ces toiles des représentations de sainte Françoise romaine pleurant sa fille, sainte Olive de Palerme tenant un sceptre et une branche d'olivier, sainte Cécile tenant un triangle et une baguette, une autre sainte martyre tenant une couronne et un sceptre et une allégorie de la modestie. Cette interprétation, non totalement satisfaisante, est néanmoins plausible, bien qu'aucun signe religieux distinctif, ni auréole, ni croix, ne vienne la conforter. Cette série porte bien la marque de Guy François : cadrage serré, fond neutre, ovale régulier des visages, regards graves, élégance des gestes des mains, et aussi exécution ferme, coloris chaud, rendu attentif des matières, surtout dans les détails. La datation de cet ensemble est difficile à déterminer, en l'absence de tout document, mais la qualité et l'homogénéité de l'ensemble suggéreraient une date antérieure à celle de la série des « Femmes fortes ». 
Figure 7

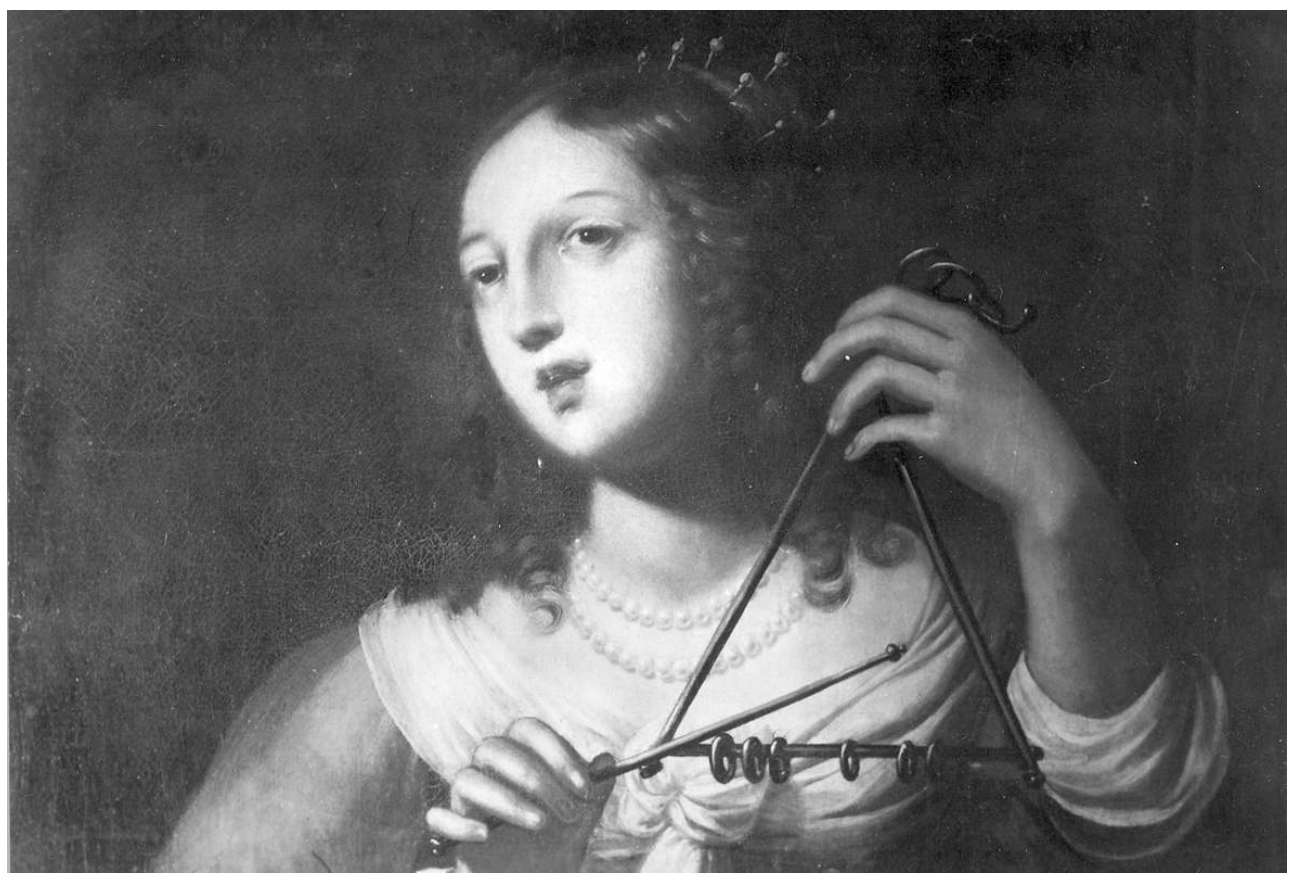

Guy François, une allégorie ou une sainte, huile sur toile, 64 cm x 92 cm, Puy-de-Dôme, Sermentizon, château d'Aulteribe, Centre des Monuments Nationaux. Bruno Saunier, 1995.

(c) Bruno Saunier.

\section{Figure 8}

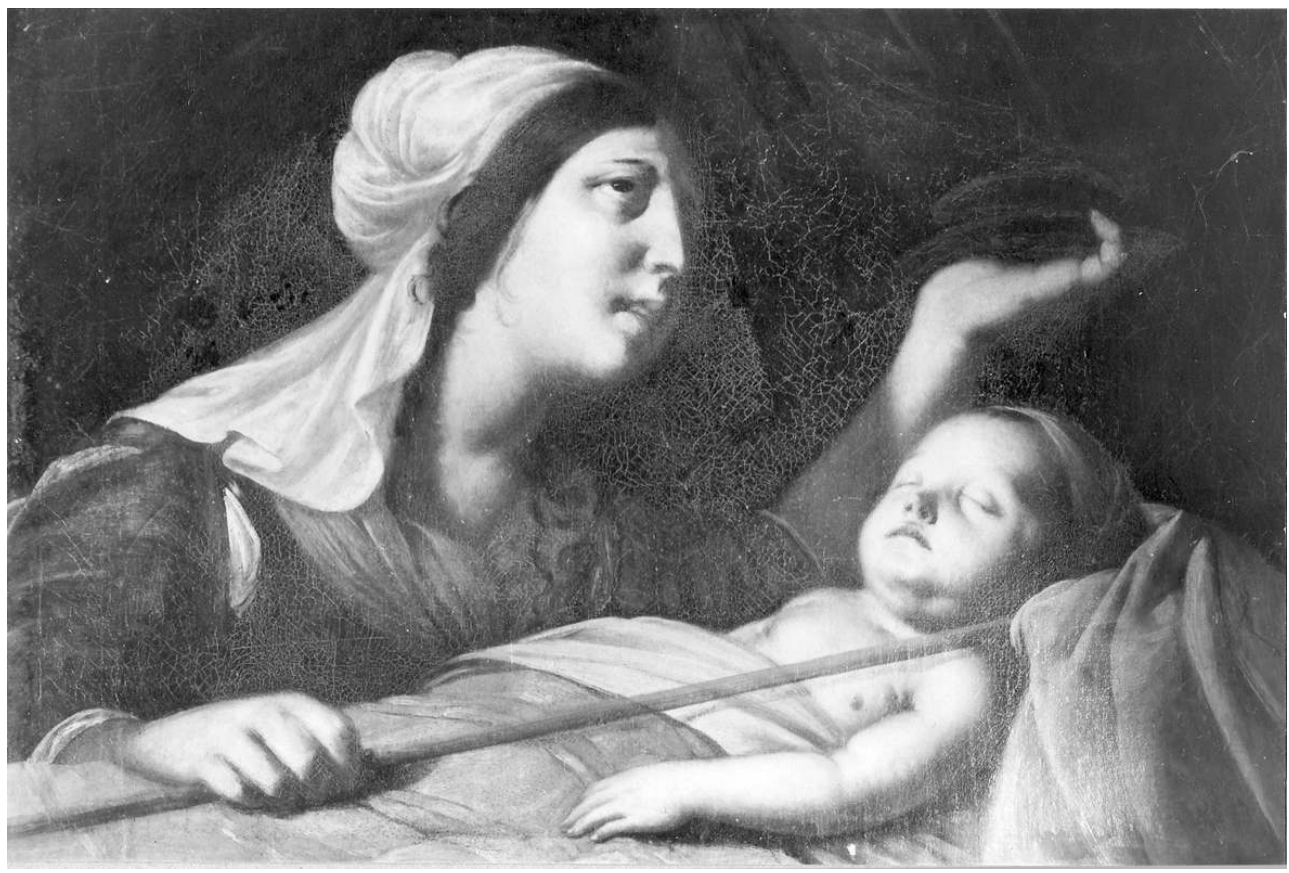

Guy François, une allégorie ou une sainte, huile sur toile, 64 cm x 92 cm, Puy-de-Dôme, Sermentizon, château d'Aulteribe, Centre des Monuments Nationaux. Bruno Saunier, 1995.

(c) Bruno Saunier. 


\section{Quel commanditaire?}

24 même de commander une suite des «Femmes illustres"? Il ne s'agit pas, on l'a vu précédemment, de tableaux anodins. Au moment où se déroule la régence difficile et contestée d'Anne d'Autriche, avoir chez soi une suite semblable constitue assurément un témoignage de fidélité à la régente, en plus d'un témoignage de grande culture et de connaissance des modes parisiennes. La provenance des tableaux n'est pas connue. La plus ancienne mention remonte à l'année 1768 où ils figurent dans un inventaire du château où ils sont encore conservés. Cet inventaire indique "À l'étage, à l'entrée d'une chambre: "cinq tableaux femmes fortes avec leurs cadres usés " ${ }^{11}$. On remarque que, dès cette époque, un sixième tableau, sans doute la Judith, est séparé des cinq autres. Cependant, cette mention de l'année 1768 n'apporte aucune certitude sur la provenance antérieure de la série. Vers 1650, le Velay, comté ressortissant de la grande province du Languedoc mais ayant ses propres États, ne compte guère de grandes familles richement possessionnées et nanties d'importants châteaux à la mode avec chambres ou galeries ornées de peintures.

personnages pourraient avoir commandé cette série des «Femmes illustres": l'évêque du Puy, Henri de Maupas, et le vicomte de Polignac, Gaspard-Armand, marquis de Chalençon. Ils sont tous les deux des clients de Guy François. Nous le savons parce que Guy a peint leur portrait. Celui d'Henri de Maupas, aujourd'hui perdu, figure dans l'inventaire après décès de Claude François, le frère cadet de Guy ${ }^{12}$ et celui de GaspardArmand est encore conservé par la famille ${ }^{13}$.

Le premier personnage est aussi le premier par rang d'importance dans le Velay. Nommé en 1641 sur le siège du Puy dont il prend possession en 1644, Henri Cauchon de Maupas du Tour est également aumônier de la reine, Anne d'Autriche, depuis $1634^{14}$. Appartenant à une importante famille champenoise alliée aux Gondi, il est proche de la régente. Le 21 avril 1643, on le voit assister au baptême du dauphin, futur Louis XIV, dans la SainteChapelle de Saint-Germain-en-Laye, aux côtés de la reine, de la princesse de Condé, de la comtesse de Soissons, de la duchesse de Longueville et du cardinal Mazarin. Il figure parmi les évêques de Beauvais, de Viviers, de Riez, de Saint-Pol et de Coutances, tandis qu'officie l'évêque de Meaux, Dominique Séguier ${ }^{15}$. Le prélat appartient au parti des dévots et il est connu pour ses liens avec Vincent de Paul et François de Sales. Auteur d'une vie de la vénérable mère Jeanne Françoise de Chantal, la future sainte Jeanne de Chantal, il a également encouragé la création, à l'initiative du jésuite le père Médaille, de la congrégation des sœurs de saint Joseph dont les religieuses n'étaient pas cloîtrées mais invitées au contraire à aller vers le monde. On peut parfaitement imaginer Mgr de Maupas commander cette série des «Femmes illustres " comme témoignage à la fois de son engagement auprès de la reine régente et de sa volonté de promouvoir la femme incarnant les «valeurs morales fondées sur des commandements divins ». Il reste sur le siège épiscopal du Puy jusqu'en 1661, année où il est transféré à Évreux.

Face à lui, l'autre personnage important du Velay est Gaspard-Armand, vicomte de Polignac, marquis de Chalençon. Il nait en 1579 et meurt en 1659. Premier baron du Velay, il est le représentant d'une lignée fort ancienne, puisqu'elle remonte au moins au IX siècle. Il est gouverneur du Puy et s'oppose souvent à l'évêque. Il fait partie de la septième promotion de chevaliers de l'Ordre du Saint-Esprit nommée par Louis XIII en 1633, 
promotion importante en nombre et en qualité puisqu'elle comptait le cardinal de Richelieu. Dans son Histoire générale du Languedoc, dom Vaissette cite à plusieurs reprises le vicomte de Polignac parmi les membres de la noblesse assistant à la tenue des États de la province. C'est lui qui aménage l'antique forteresse de La Voûte dans les gorges de la Loire, non loin du Puy, et fait construire le portail à bossages dans l'axe de l'entrée du château. Dans son testament, Guy François nomme le vicomte de Polignac parmi ses créanciers. On peut donc imaginer que notre peintre a exécuté plusieurs tableaux pour la famille de Polignac, outre le portrait du chef de maison. Il serait difficile, en effet, d'imaginer que le vicomte n'ait toujours pas payé le montant dû pour son portrait quelque vingt années après son exécution.

Cependant, aujourd'hui, aucune preuve, aucun document ne permet de pencher en faveur de l'un ou l'autre de ces deux personnages.

\section{Conclusion}

Cette série des « Femmes illustres » ou «Femmes fortes » montre donc un Guy François différent de ce que l'on connaissait jusqu'à présent. Elle révèle un peintre moins isolé qu'il a souvent été écrit, capable de créer de nouvelles œuvres sur des sujets nouveaux, jusqu'à la fin de sa carrière, et connaissant les modes et la culture savante parisiennes. De fait, l'artiste a longtemps été estimé, bien au-delà de son Velay natal, et les Salons toulousains du XVIII ${ }^{e}$ siècle montrent que ses œuvres étaient toujours collectionnées et appréciées. Il reste encore à découvrir portraits et scènes mythologiques mentionnés dans les archives et qui doivent dormir dans quelque grenier de château. Il n'en demeure pas moins un peintre de la réalité, caravagesque par certains traits, mais en même temps proche de la peinture bolonaise des Carrache et de Guido Reni. Jusque dans le traitement de ces "Femmes illustres", Guy François conserve ce style inimitable, ancré dans l'observation du quotidien.

\section{NOTES}

1. Guy François (1578 ?-1650), catalogue rédigé par Marie-Félicie Perez. Le Puy-en-Velay, musée Crozatier, 22 juin- $1^{\text {er }}$ septembre, Saint-Étienne, musée d'Art et d'industrie, 20 septembre-31 octobre 1974.

2. SCHLODER, J. E. «Un artiste oublié : Nicolas Prévost, peintre de Richelieu », Bulletin de la Société de l'Histoire de l'Art Français, séance du 5 janvier 1980, p. 65, illustrations n 5 à 11 . Catalogue de l'exposition Vouet. Paris: Galeries nationales du Grand Palais, 6 novembre 1990-11 février 1991, p. 136. BREJON DE LAVERGNÉE, B., de REYNIÈS, N., SAINTE FARE GARNOT, N. Charles Poerson, 1609-1667. Paris : éditions Arthena, 1997, p. 100-105, illustration $\mathrm{n}^{\circ} 33$. PACHT BASSANI, P. Claude Vignon, 1593-1670. Paris: éditions Arthena, 1992, p. 437-448, illustrations $n^{\circ} 434 G$ à 454 . de REYNIÈS, N., LAVEISSIÈRE, S. Isaac Moillon (1614-1673) un peintre du roi à Aubusson. Paris : éditions Somogy, 2005, p. 280-297, illustrations n X.1 à X.7.b. 
3. DENIS, I. «Henri Lerambert et l'Histoire d'Artémise ", actes du colloque international de Chambord, La tapisserie au xvii ${ }^{\mathrm{e}}$ siècle et les collections européennes, 18 et 19 octobre 1996. Paris : éditions du Patrimoine, 1999, p. 33-50.

4. PASCAL, C. "Les recueils de femmes illustres au xvii ${ }^{e}$ siècle ", actes du colloque, Connaître les femmes de l'Ancien Régime. Paris : Société Internationale pour l'Étude des Femmes de l'Ancien Régime (SIEFAR).

5. GIRON, L. « Son fils, Jean François, continua les errements de son père qu'il copia ou imita, mais sans avoir ni son style, ni son élévation, ni sa vigueur de coloris, ni sa hardiesse d'exécution. La ligne et l'anatomie n'existent plus chez lui. Il s'en tient, pour les chairs, à de molles rondeurs d'un ton doré et avec une application de vermillon sur les joues; ses têtes ont toutes le même caractère familial. Du reste, il se complaisait à peindre des personnages féminins auxquels ces rondeurs et ces vermillons n'allaient point trop mal. J'ai vu de lui, entre autres, quelques viragos empruntées aux estampes de la Galerie des femmes fortes du Père Lemoyne, Semiramis, Cléopâtre etc., qui sont des portraits décoratifs sans valeur historique ou artistique.» («Le peintre Guy François du Puy », Réunion des sociétés des Beaux-Arts des départements, 1897, p. 546-556).

6. Inventaire après décès de Claude François, marchand, 11 janvier 1665, Archives départementales de la Haute-Loire, série $3^{\mathrm{E}} 290-1$, folios 162 et suivants.

7. PEPPER, S. Guido Reni, l'opera completa. Novara : Istituto geografico De Agostini, 1988, n - 134, p. 277, illustration 125, et n. 22, p. 333, illustration 16.

8. Les registres de baptême du baptistère Saint-Jean du Puy ont disparu pour cette période mais d'autres documents laissent supposer une naissance en 1578.

9. Archives départementales de la Haute-Loire, série $3^{\mathrm{E}} 290-1$, folios 52 et suivants.

10. Ces tableaux de dimensions semblables (H.: $64 \mathrm{~cm}$; L.: $92 \mathrm{~cm}$ ) ont été signalés par M. PETTEX-SABAROT. «Guy François : attributions et propositions à propos d'un ensemble de toiles franciscaines ", Revue du Louvre et des Musées de France, n 5-6, 1979, p. 414-426. Ils avaient été attribués à Guy François par Jacques Foucart dès 1977 (cf. catal. expos. «Le siècle de Rubens dans les collections publiques françaises ». Paris : Galeries nationales du Grand Palais, 1977-1978, p. 54, $\mathrm{n}^{\circ} 19$, notice d'un tableau de Jean Brueghel de Velours conservé au château d'Aulteribe). Leur provenance ancienne n'est pas connue, avant le legs à la Caisse nationale des Monuments historiques du château et de ses collections par le marquis et la marquise de Pierre en 1960. On peut noter, cependant, que les Pierre avaient rassemblé à Aulteribe le contenu de pas moins quatre demeures dont, notamment, une partie du mobilier du château de Sistrières dans le Cantal dont ils avaient hérité de la famille de Murat-Sistrière. Ces six tableaux pourraient peutêtre provenir de ce dernier château.

11. L'original de cet inventaire après décès est conservé dans une collection privée du Puy-enVelay mais les propriétaires actuels du château où se trouvent les tableaux en conservent une copie. Il avait été dressé en 1768 au moment du décès de M. de Veyrac de Maisonseule.

12. Archives départementales de la Haute-Loire, série $3^{\mathrm{E}}$ 290-1 fol. 162 et suivants : «Plus deux tableaux l'un représentant un saint Jean Baptiste et un agneau avec un cadre, et l'autre le portrait du seigneur de Maupas, evesque du Puy, estimés trois livres ».

13. Tableau inédit, huile sur toile, $75 \mathrm{~cm} \times 65 \mathrm{~cm}$ environ.

14. CUBIZOLLES, P. Le diocèse du Puy des origines à nos jours. Nonette : éditions CRÉER, 2005, p. 325-326.

15. HILDESHEIMER, Fr. La double mort du roi Louis XIII. Paris : Flammarion, 2007, p. 184 : « Le jeune garçon, «beau comme un ange », pénètre dans l'église vêtu par-dessus son habit ordinaire d'une robe de taffetas d'argent. Il est suivi de sa gouvernante, Mme de Lansac. Puis, paraît la reine, accompagnée de la princesse de Condé, de la comtesse de Soissons, de la duchesse de Longueville et du cardinal Mazarin. Sont encore présents les évêques de Beauvais, de Viviers, de Riez, de Saint-Pol, de Coutances et du Puy. » 


\section{RÉSUMÉS}

Guy François (vers 1578-1650) est le principal peintre religieux de l'Auvergne et du Languedoc pendant la première moitié du XVII ${ }^{\mathrm{e}}$ siècle. Artiste caravagesque, il peint de grandes toiles pour des retables mais aussi des tableaux de dévotion privée et des décors. La découverte d'une série de toiles des « Femmes illustres » conservée dans une propriété de la Haute-Loire montre l'artiste sous un nouveau jour. En outre, ces toiles apportent un éclairage nouveau sur la culture savante dans le petit comté du Velay au cours du XVII e siècle et sur les rapports avec la mode et les événements parisiens du temps de la régence d'Anne d'Autriche.

Guy François (c. 1578-1650) is probably the most important religious painter in Auvergne and Languedoc during the first half of the $17^{\text {th }}$ century. Member of the caravagesque movement, he painted large canvas for churches but also small paintings for private devotion. A set of six canvas about the "Femmes illustres " was rediscovered in a castle near Le Puy-en-Velay and gives a new dimension of his work. They also bring us a new perspective about the cultural life of the little county, le Velay, in relation with the Parisian fashion and political events during Anne d'Autriche's regency.

\section{INDEX}

Keywords : painting

Mots-clés : Marie Stuart, Panthée, Lucrèce, Porcia, Judith, Zénobie, Polignac, Maupas (Henri de), Anne d'Autriche, Caravagesque, Peinture, François (Guy) ou Guy François, Velay, Haute-Loire, femmes fortes, femmes illustres

\section{AUTEURS}

\section{MARIE-FÉLICIE PEREZ}

Professeur d'histoire de l'art émérite, université Louis Lumière, Lyon II. mfpp@wanadoo.fr

\section{BRUNO SAUNIER}

Conservateur en chef du patrimoine, Direction des musées de France.

bruno.saunier@culture.gouv.fr 